\title{
Amyloid Resorption Induction
}

National Cancer Institute

\section{Source}

National Cancer Institute. Amyloid Resorption Induction. NCI Thesaurus. Code C41566.

Amyloid Resorption Induction involves initiation of activities involved in the breakdown and assimilation of amyloid deposits. 